\title{
GLOBAL DRIFT WAVES IN WEAKLY IONIZED PLASMA COLUMN
}

\author{
P. P. Sosenko ${ }^{1,2}$, M. Poleni ${ }^{1}$ \\ ${ }^{1}$ Université Henri Poincaré - Nancy I, LPMIA, \\ BP 239, 54506 Vandoeuvre les Nancy, Cedex, France \\ ${ }^{2}$ International Centre of Physics \& M. M. Bogoliubov Institute for Theoretical Physics \\ of the National Acad. Sci. of Ukraine, \\ 146 Metrolohichna Str., Kyiv, 03143, Ukraine
}

(Received June 18, 2002)

\begin{abstract}
The temperature gradient effect on global drift waves in cylindrical weakly ionized plasmas is investigated. The important role of the temperature gradient in plasma oscillation properties, like eigenfrequencies, instability thresholds and rates, mode localization, is shown.

Key words: weakly ionized plasma, drift wave, instability threshold.
\end{abstract}

PACS number(s): 52.35.Kt, 52.35.Fp

\section{INTRODUCTION}

The important role of drift waves is well-known, and they continue to be the subject of intense investigation (fusion plasma, astrophysical situations, ionosphere applications, plasmas in laboratory devices, such as Mirabelle in France, and KIWI in Germany [1-4]). The drift-wave frequencies are much less than the ion cyclotron frequency $\Omega_{\mathrm{i}}$. Such waves make an example of plasma motion similar to the motion of ordinary fluid, when turbulence and anomalous transport can occur. Therefore, the drift approximation [5] becomes inevitable.

In this article, we study the drift-wave instabilities in the situations [1, 3-4] of low-pressure (argon) cylindrical plasma column immersed in the axial magnetic field, and in the presence of macroscopic density and temperature gradients. In this regard we extend and generalize previous studies [6-7], carried out in the approximation of constant plasma temperature. The latter is not usually satisfied under experimental conditions. We show the important role of the temperature gradient in plasma oscillation properties (eigenfrequencies, instability thresholds and rates, mode localization). For arbitrary temperature and density profiles, general theoretical considerations relying on quantum-mechanical analogies, integral constraints, and numerical methods can be applied.

The article structure and results are as follows. In Section 2 the basic eigen-value problem is posed. In Section 3 a quantum-mechanical analogy is used to establish a general dispersion equation for the low-frequency cylindrical waves, and to explain possible temperaturegradient effects in the general case of arbitrary temperature profiles. It is argued that the radial temperature variation can govern the radial mode structure and localization, as well as the eigenfrequency. The temperature inhomogeneity can be more important than the density one, since the density inhomogeneity enters via the logarithmic derivative only, which has a weak spatial variation. Normal temperature profiles play the role of a narrow "effective potential well" for the waves. For larger temperature gradients, in the case of normal equilibrium profiles, the plasma modes are localized more and closer to the plasma centre. Then the effect of charge separation near the plasma edge becomes less important. In this way, the "localization paradox" of previous studies can be removed. On the other hand, in the presence of temperature gradient, the eigenfrequency becomes considerably lower. In this case, the validity conditions for the low-frequency approximation become less stringent. In Section 4, integral constraints are derived in the general case of arbitrary density and temperature profiles. Such constraints make it possible to evaluate, or to find analytically/numerically the eigenfrequencies, the instability thresholds and rates for complicated realistic conditions, and for various models of electron density response. In Section 5, a particular case of Lorentzian temperature profile is considered. The eigen-value problem is reduced to the two-dimensional quantum oscillator with the harmonic potential-well bottom. The latter problem is solved analytically, and the solution is related to the one in the case of homogeneous temperature, but with renormalized parameters. The radial mode structure of the cylindrical waves and the drift-wave eigenfrequency are found. The limits of radially infinite/finite systems are introduced.

\section{BASIC EQUATION}

The basic eigen-value equation for the global drift waves [8] is

$$
\left(\triangle_{\perp}+\kappa_{n 0} \cdot \nabla_{\perp}\right) \hat{\phi}=-\left(\frac{\omega_{*}}{\omega+\nu_{\text {in }}}-\tilde{\chi}_{e}\right) \hat{\phi}
$$

in the quasi-neutral approximation (when charge separation effects are neglected: $\delta n_{e}=Z_{\mathrm{i}} \delta n_{\mathrm{i}}$ ), and when the ion motion along the magnetic field is disregarded. This equation is derived within the context of plasma fluid description $[5,9]$, for the perturbations of an equilibrium without mean electric fields.

Here, the dimensionless variables are used, with the scales $\Omega_{\mathrm{i}}=q_{\mathrm{i}} B / m_{\mathrm{i}} c$ for the frequency, $\rho_{\mathrm{s}}=c_{\mathrm{s}} / \Omega_{\mathrm{i}}$ for the 
space coordinates $\left(c_{\mathrm{s}}^{2}=Z_{\mathrm{i}} \bar{T} / m_{\mathrm{i}}\right), \bar{n}$ for the density, $\bar{T}$ for the temperature, and the dimensionless electric field potential is $\phi=e \Phi / \bar{T}, q_{\mathrm{i}}=Z_{\mathrm{i}} e$ and $m_{i}$ are the ion charge and mass, $e$ is the absolute value of the electron charge, $m_{e}$ is the electron mass, $B$ is for a constant magnetic field, $c$ is the light velocity in the vacuum, $\nu_{\text {in }}$ is the ion-neutral collision frequency.

In cylindrical geometry, with the magnetic field along $z$ and the density and temperature gradients in the radial direction, the potential perturbation is represented as follows

$$
\phi \equiv \sum_{\omega} \sum_{k_{\|}} \sum_{l=-\infty}^{\infty} \exp \left(i l \theta+i k_{\|} z-i \omega t\right) \hat{\phi}\left(l, k_{\|}, \omega, r\right)
$$

where $\hat{\phi}$ is a cylindrical wave amplitude, $l$ is an azimuthal mode number, and the frequency summation is over all the eigenfrequencies $\omega\left(l, k_{\|}\right)$. Other wave quantities are represented in a similar manner. $\triangle_{\perp}+\kappa_{n 0} \cdot \nabla_{\perp} \equiv$ $\frac{1}{r} \frac{d}{d r} r \frac{d}{d r}-\frac{l^{2}}{r^{2}}+\kappa_{n 0} \frac{d}{d r}, \kappa_{n 0}=d \ln n_{e 0} / d r$.

The density gradient effects enter with a non-vanishing logarithmic derivative, $\kappa_{n 0} \neq 0$. The Gaussian density profile $n_{0} \sim \exp \left(-r^{2} / L_{N}^{2}\right)$ is a good approximation for many experimental situations. The term $\kappa_{n 0} \cdot \nabla_{\perp} \hat{\phi}$ is the contribution from the ion polarization drift. It can be essential for global/large-scale oscillations with $\kappa_{n 0} \sim \nabla_{\perp}$. The response function relates the density and potential perturbations [8], $\delta \hat{n}_{e} / n_{e 0}=\tilde{\chi}_{e} \delta \hat{\phi}$,

$$
\tilde{\chi}_{e}=\frac{\omega_{*}+i \Gamma_{\|} / \tau}{\omega-\omega_{U}+i \Gamma_{\|}}
$$

with the density-gradient-driven drift frequency $\omega_{*}=$ $-l \kappa_{n 0} / r$, the dimensionless temperature profile $\tau=$ $T_{e} / \bar{T}$, the frequency shift in the presence of axial electron flow $\omega_{U}=\bar{U} k_{\|} \sqrt{M / Z_{\mathrm{i}}}$, where $\bar{U}=U / S_{\mathrm{e}}$ is the ratio of parallel drift velocity and parallel thermal velocity for electrons, $\Gamma_{\|}=D_{e} k_{\|}^{2}$ is the rate of electron diffusion along the magnetic field, $D_{e}=\tau M / Z_{\mathrm{i}} \nu_{\text {en }}$ is a dimensionless diffusion coefficient $\left(D_{e}=S_{e}^{2} / \nu_{e n}\right.$, in dimensional units), $M=m_{\mathrm{i}} / m_{e}$, and $\nu_{e n}$ is an electron-neutral collision frequency. If the electron inertia is important, then $\nu_{e n}$ in $\Gamma_{\|}$is replaced with $\nu_{e n}-i\left(\omega-\omega_{U}\right)$. The electron electric susceptibility $\chi_{e}(\mathbf{k}, \omega)$ in the local theory is related to $\tilde{\chi}_{e}: \chi_{e}(\mathbf{k}, \omega)=\tilde{\chi}_{e} / k^{2} \lambda_{e}^{2}$ in dimensional units, with $\lambda_{e}^{2}=T_{e} / 4 \pi e^{2} \bar{n}_{e 0}$.

When $\Gamma_{\|}$is much larger than all other frequencies in $\tilde{\chi}_{e}$, then the electron density perturbation is governed approximately by the linearized Boltzmann's law,

$$
\tilde{\chi}_{e}=\frac{1}{\tau}(1+i \delta), \delta=\frac{1}{\Gamma_{\|}}\left(\omega-\omega_{U}-\tau \omega_{*}\right) .
$$

Small deviations from the Boltzmann's law, described by $\delta$, determine possible mechanisms of weak instability [9].

The electron-temperature gradient is taken into ac- count via the dimensionless temperature profile $\tau\left(\mathbf{r}_{\perp}\right)=$ $T\left(\mathbf{r}_{\perp}\right) / \bar{T}$ contained in $\tilde{\chi}_{e}$.

\section{QUANTUM-MECHANICAL ANALOGY}

The substitution $\hat{\phi}=\tilde{\phi} / \sqrt{n_{e}}$ transforms the wave equation into

$$
\left[-\frac{1}{2} \triangle_{\perp}+V(\mathbf{r})\right] \tilde{\phi}=\frac{1}{2} K^{2} \tilde{\phi}
$$

with an effective potential $V(\mathbf{r})=\frac{1}{4}\left(\nabla \cdot \kappa_{n 0}+\frac{1}{2} \kappa_{n 0}^{2}\right)+$ $\frac{1}{2}\left(\tau^{-1}-1\right)$, and $K^{2} \equiv \omega_{*} / \omega-1$, in the ideal case, $\nu_{\text {in }}=0$, and for the adiabatic electrons, $\tilde{\chi}_{e}=1 / \tau$. In the case of Gaussian density profile, $n_{0} \sim \exp \left(-r^{2} / L_{N}^{2}\right)$, $\omega_{*}$ is constant, and the wave equation reduces to a twodimensional Schrödinger's equation

$$
\left[-\frac{1}{2} \triangle_{\perp}+\frac{1}{2} \tilde{r}^{2}+\frac{1}{2} L_{N}^{2}\left(\tau^{-1}-1\right)\right] \tilde{\phi}=E \tilde{\phi}
$$

Here $\tilde{\mathbf{r}}=\mathbf{r} / L_{N}$ is a new dimensionless space variable, and the "energy" eigen-value is related to the eigenfrequency:

$$
E=1+\frac{1}{2}\left(L_{N} K\right)^{2}=1+\frac{1}{2}\left(\omega_{*} / \omega-1\right) \geq 1 .
$$

The energy is greater than unity because $K^{2}$ is real and positive as an eigen-value of the hermitian Hamiltonian with $V_{\min } \geq 0$ (one can always choose the temperature scale $\overline{\bar{T}}$ to ensure the latter inequality), therefore $|\omega| \leq\left|\omega_{*}\right|$. The unity corresponds formally to the energy of the "2-D harmonic-oscillator vacuum state", and there is a discrete energy spectrum. The latter equation yields the dispersion relation, similar to the one in the case of homogeneous temperature: $\omega=\frac{\omega_{*}}{1+K^{2}}$.

The Schrödinger form of the eigen-value equation enables one to understand possible temperature-gradient effects in the general case of arbitrary temperature profiles. The density inhomogeneity enters via the logarithmic derivative only, which has a weak spatial variation. Therefore, the radial temperature variation can govern the radial mode structure and localization, as well as the eigenfrequency. For monotonic temperature dependence (with the maximal value $\tau=1$ at the plasma centre), a modulated well bottom becomes deeper and narrower when the temperature gradient increases, then the energy levels rise higher. Therefore, for larger temperature gradients, $K^{2}$ increases and the eigenfrequency decreases. This conclusion explains one possible reason why the low-frequency oscillations can dominate under experimental conditions. With temperature inhomogeneity being taken into account, the validity condition for the low-frequency approximation becomes less stringent. 
The mode localization is affected as well. For larger temperature gradients, in the case of normal equilibrium profiles, the plasma modes are localized more and closer to the plasma centre. Then the effects of charge separation near the plasma edge become less important. Another interesting observation is that the temperaturegradient effects are more pronounced when the ratio $L_{N}^{2} / \rho_{\mathrm{s}}^{2}$ is larger (i.e. for stronger magnetic fields, weaker density gradients, smaller temperature, smaller ion mass, and larger ion charge).

In cylindrical geometry, $\hat{\phi} \propto r^{l}$, when $r \rightarrow 0$, for arbitrary density and temperature profiles if the density gradient is finite at $r=0$. In order to find the behaviour of the potential when $r \rightarrow \infty$, we reduce as usual the eigen-value equation to the one-dimensional Schrödinger's equation by changing the unknown function $\hat{\phi}=Y / \sqrt{r n_{e 0}}$ :

$$
Y^{\prime \prime}=\left(\frac{l^{2}-\frac{1}{4}}{r^{2}}+2 V-K^{2}\right) Y .
$$

In the case of Gaussian density profile with $\kappa_{n 0}=$ $-2 \mathbf{r} / L_{N}^{2}$, the interesting evanescent solution is

$$
\begin{aligned}
& \hat{\phi}=Y / \sqrt{r n_{e 0}} \sim \exp \left(\frac{r^{2}}{2 L_{N}^{2}}\right. \\
& \left.-\int d r \sqrt{\frac{r^{2}}{L_{N}^{4}}+\frac{1}{\tau}-1}\right), r \rightarrow \infty .
\end{aligned}
$$

We stress that the solution evanescent at infinity exists if only the temperature inhomogeneity is retained.

In the limit of $1 / \tau \ll r^{2} / L_{N}^{4}, \hat{\phi} \sim \exp \left(-\int d r \frac{L_{N}^{2}}{2 r \tau}\right)$.

For example, if $1 / \tau \sim r^{a}, 0<a<2$, then $\hat{\phi} \sim$ $\exp \left(-\frac{L_{N}^{2}}{2 a \tau}\right)$

In the opposite limit of $1 / \tau \gg r^{2} / L_{N}^{4}$, if $1 / \tau \sim r^{a}$, $a>2$, then $\hat{\phi} \sim \exp \left(-\frac{2 r \sqrt{\tau^{-1}}}{a+2}\right)$.

If $1 / \tau \sim r^{2} / L_{T}^{2}$, where $L_{T}$ is a characteristic temperature-gradient scale, then $\hat{\phi} \sim$ $\exp \left[\frac{r^{2}}{2 L_{N}^{2}}\left(1-\sqrt{1+L_{N}^{2} \eta^{2}}\right)\right]$, where $\eta \equiv L_{N} / L_{T}$ is the ratio between the density and temperature gradient scales. In the particular case of Lorentzian temperature profile, an exact analytical solution can be found (see below).

\section{INTEGRAL CONSTRAINTS}

The eigen-value equation yields the following integral constraint

$$
\int n_{0}|\nabla \hat{\phi}|^{2} d \mathbf{r}=\int n_{0}|\hat{\phi}|^{2} \frac{\omega_{*}-\omega \tilde{\chi}_{e}}{\omega+i \nu_{\text {in }}} d \mathbf{r}
$$

It is derived after multiplying the eigen-value equation by $n_{0} \hat{\phi}^{*}$ and integrating by parts for zero boundary conditions. In the ideal limit and in the case of adiabatic electrons, $\omega=\frac{1}{W} \int n_{0} \omega_{*}|\hat{\phi}|^{2} d \mathbf{r}$, where $W=W_{\mathrm{K}}+W_{\mathrm{P}}=$ $\int n_{0}|\nabla \hat{\phi}|^{2} d \mathbf{r}+\int \frac{n_{0}}{\tau}|\hat{\phi}|^{2} d \mathbf{r}$. The quantity $W$ corresponds to the total oscillation energy, while $W_{\mathrm{k}} \equiv \int n_{0}|\nabla \hat{\phi}|^{2} d \mathbf{r}$, and $W_{\mathrm{P}} \equiv \int \frac{n_{0}}{\tau}|\hat{\phi}|^{2} d \mathbf{r}$ can be associated with the particle kinetic energy of electric-drift motion (the polarizationdrift contribution is negligible), and the potential energy, respectively. For monotonic density profiles, $\omega$ has the sign of the density-gradient drift frequency $\omega_{*}$.

We use also the following notations for mean values

$$
\begin{aligned}
& \langle Q\rangle \equiv \frac{\int n_{0} Q|\hat{\phi}|^{2} d \mathbf{r}}{\int n_{0}|\hat{\phi}|^{2} d \mathbf{r}}, \\
& k^{2} \equiv \frac{\int n_{0}|\nabla \hat{\phi}|^{2} d \mathbf{r}}{\int n_{0}|\hat{\phi}|^{2} d \mathbf{r}} \text { ('mean squared gradient value'). }
\end{aligned}
$$

Then the eigenfrequency is $\omega_{r}=\left\langle\omega_{*}\right\rangle /\left(k^{2}+\left\langle\tau^{-1}\right\rangle\right)$. If the temperature scale $\bar{T}$ is chosen as the maximal temperature value, then $\left\langle\tau^{-1}\right\rangle>1$ by definition. When $\tau=1$, the mean squared gradient value is the ratio of kinetic and potential energies, $k^{2}=W_{\mathrm{K}} / W_{\mathrm{P}}$.

If ion-neutral collisions are taken into account, the oscillations appear to be damped:

$$
\omega=\frac{\left\langle\omega_{*}\right\rangle-i \nu_{\text {in }} k^{2}}{k^{2}+\left\langle\tau^{-1}\right\rangle}
$$

In the general case,

$$
\omega=\frac{\left\langle\omega_{*}\right\rangle-i \nu_{\text {in }} k^{2}}{k^{2}+\left\langle\tilde{\chi}_{e}\right\rangle} .
$$

This expression can be simplified in the case of weak ion-neutral collisions, when $\nu_{\text {in }} \ll \omega$, and weak deviations from the adiabatic electron-density response, when $\tilde{\chi}_{e}=\frac{1}{\tau}(1+i \delta)$, with $|\delta| \ll 1$, when the imaginary part $\omega_{i}$ of the frequency $\omega=\omega_{r}+i \omega_{\mathrm{i}}$ is small (transparency domain),

$$
\left|\omega_{\mathrm{i}} / \omega_{r}\right| \ll 1, \omega_{r}=\left\langle\omega_{*}\right\rangle /\left(k^{2}+\left\langle\tau^{-1}\right\rangle\right) .
$$

One can find by iterations:

$$
\omega_{\mathrm{i}}=-\nu_{\mathrm{in}} \frac{W_{K}}{W}-\frac{\omega_{r}}{W} \int \operatorname{Re} \delta \frac{n_{0}}{\tau}|\hat{\phi}|^{2} d \mathbf{r}
$$

Then the necessary instability condition is $\omega_{r} \int \operatorname{Re} \delta \frac{n_{0}}{\tau}|\hat{\phi}|^{2} d \mathbf{r}<0$ 
As $\left|\omega_{\mathrm{i}} / \omega_{r}\right| \ll 1$, one can compute $W_{\mathrm{K}}, W_{\mathrm{P}}$ and $\omega_{\mathrm{i}}$ from the eigen-function and its gradient $\left(|\hat{\phi}|^{2}\right.$ and $\left.|\nabla \hat{\phi}|^{2}\right)$ or from the eigen-function and $\omega_{r}$ obtained in the limit $\delta=0$. Experimentally observed temperature and density profiles can be taken into account in such computations.

In the fluid model under consideration, $\operatorname{Re} \delta=$ $\frac{1}{\Gamma_{\|}}\left(\omega_{r}-\omega_{U}-\tau \omega_{*}\right)$. The imaginary part of the frequency is represented as the sum $\omega_{\mathrm{i}}=\gamma_{\text {in }}+\gamma_{\text {dis }}+\gamma_{U}$, where

$$
\gamma_{\text {dis }}=\frac{\omega_{r}^{2}}{\bar{\Gamma}_{\|}\left\langle\omega_{*}\right\rangle}\left[\left\langle\tau^{-1} \omega_{*}\right\rangle-\omega_{r}\left\langle\tau^{-2}\right\rangle\right]
$$

is the drift-dissipative instability rate without axial drift,

$$
\gamma_{U}=\frac{\omega_{r}^{2} \omega_{U}}{\bar{\Gamma}_{\|}\left\langle\omega_{*}\right\rangle}\left\langle\tau^{-2}\right\rangle
$$

is the axial-drift correction to the instability rate,

$$
\gamma_{\text {in }}=-\nu_{\text {in }}\left(1-\omega_{r} \frac{\left\langle\tau^{-1}\right\rangle}{\left\langle\omega_{*}\right\rangle}\right)
$$

is the dissapation rate owing to ion-neutral collisions, where $\bar{\Gamma}_{\|}=D_{\mathrm{e}} k_{\|}^{2} / \tau$ is introduced as a space independent quantity. In the case of Gaussian density profile in cylindrical geometry, $\left\langle\omega_{*}\right\rangle=\omega_{*}=$ const.

\section{LORENTZIAN TEMPERATURE PROFILE}

We take the Gaussian density profile, $n_{0} \sim$ $\exp \left(-r^{2} / L_{N}^{2}\right)$, and the Lorentzian temperature profile of scale $L_{T}: \tau=\left(1+r^{2} / L_{T}^{2}\right)^{-1}=\left(1+\eta^{2} \tilde{r}^{2}\right)^{-1}$, where $\eta \equiv L_{N} / L_{T}$ is the ratio between the characteristic density and temperature inhomogeneity scales, and $\tilde{r}=r / L_{N}$. Then the Schrödinger's equation takes the form

$$
\left[-\frac{1}{2} \triangle_{\perp}+\frac{1}{2} \tilde{r}^{2}\left(1+L_{N}^{2} \eta^{2}\right)\right] \tilde{\phi}=E \tilde{\phi}
$$

This is the two-dimensional harmonic oscillator with the frequency $\omega_{0}=\sqrt{1+L_{N}^{2} \eta^{2}}$. The drift-wave eigenfrequency is

$$
\omega=\frac{\omega_{*}}{1+2(E-1) L_{N}^{2}}, \delta=0, \nu_{\text {in }}=0 .
$$

The quantum-mechanical result for an infinite system is $E^{(\infty)}=\left(n_{1}+n_{2}+1\right) \sqrt{1+L_{N}^{2} \eta^{2}}$ (quantization in rectangular coordinates), or $E^{(\infty)}=$ $(l+2 n+1) \sqrt{1+L_{N}^{2} \eta^{2}}$ (quantization in cylindrical coordinates). The "energy" levels rise as $\eta$ increases, therefore the eigenfrequency decreases when $\eta$ increases:

$$
\omega=\frac{\omega_{*}}{1+2\left(E^{(\infty)}-1\right) L_{N}^{2}} .
$$

In the limit, which corresponds to flat density regimes in fusion situations,

$$
\omega=\frac{\omega_{*}}{1+2(l+2 n+1) / L_{T}}, \text { when } L_{N}^{2} \eta^{2} \gg 1
$$

(or $L_{N}^{4} \gg \rho_{s}^{2} L_{T}^{2}$ in dimensional units).

The substitution $\tilde{\phi}=\hat{\phi}_{\eta} \exp \left(-\tilde{r}^{2} \sqrt{1+L_{N}^{2} \eta^{2}} / 2\right), \tilde{\mathbf{r}}=$ $\hat{\mathbf{r}} / \sqrt[4]{1+L_{N}^{2} \eta^{2}}$ gives the eigen-value equation in the form without temperature gradient,

$$
\left[\triangle_{\perp}+\left(\nabla \ln \left(-\tilde{r}^{2}\right)\right) \cdot \nabla_{\perp}\right] \hat{\phi}_{\eta}=-K_{\eta}^{2} L_{N}^{2} \hat{\phi}_{\eta}
$$

where the temperature-gradient scale enters via the constant

$K_{\eta}^{2} \equiv \frac{1}{\sqrt{1+L_{N}^{2} \eta^{2}}}\left(\frac{\omega_{*}}{\omega}-1+2 \frac{1-\sqrt{1+L_{N}^{2} \eta^{2}}}{L_{N}^{2}}\right)$.

Therefore, the solution is

$$
\hat{\phi}_{\eta} \sim \hat{r}^{l} e^{i l \theta} M\left(\frac{1}{4}\left(2 l-K_{\eta}^{2} L_{N}^{2}\right), l+1, \hat{r}^{2}\right)
$$

where $M(a, b, z)$ is the confluent hypergeometric function [10],

$$
\begin{aligned}
M(a, b, z) & =\sum_{n=0}^{+\infty} \frac{\Gamma(a+n)}{\Gamma(a)} \frac{\Gamma(b)}{\Gamma(b+n)} \frac{z^{n}}{n !} \\
& =1+\frac{a}{b} z+\frac{a(a+1)}{b(b+1)} \frac{z^{2}}{2}+\ldots
\end{aligned}
$$

convergent for any finite $z$ if $b \neq 0,-1,-2,-3, \ldots$ and where $\Gamma$ is the Euler function. The potential eigenfunctions and eigenfrequencies follow

$$
\begin{aligned}
& \hat{\phi} \propto r^{l} \exp \left(r^{2} \frac{1-\sqrt{1+L_{N}^{2} \eta^{2}}}{2 L_{N}^{2}}\right) \\
& \times M\left(\frac{1+l-E_{\eta}}{2}, l+1, r^{2} \frac{1-\sqrt{1+L_{N}^{2} \eta^{2}}}{L_{N}^{2}}\right), \quad \text { (25) } \\
& E_{\eta} \equiv \frac{E}{\sqrt{1+L_{N}^{2} \eta^{2}}}, \omega=\frac{\omega_{*}}{1+2(E-1) / L_{N}^{2}}=\frac{l}{L_{N}^{2} / 2+E-1} . \\
& \text { If } \eta \neq 0, \text { one can consider the limit of infinite } \\
& \text { system. Then the confluent hypergeometric function } \\
& M \text { must be a finite polynomial of } r^{2} \text { in order to in- } \\
& \text { crease slower than the exponential, when } r \rightarrow \infty \text {. This } \\
& \text { is achieved when } \frac{1+l-E_{\eta}}{2}=-n \Rightarrow E_{\eta}=l+2 n+1 \text {. }
\end{aligned}
$$


For a radially finite system, the boundary condition of zero potential perturbation on the conducting cylinder yields the dispersion equation $M(a, l+1, z)=0$, $E_{\eta}=l+1-2 a_{l n}\left(z_{\eta}\right), z_{\eta} \equiv \frac{R_{0}^{2}}{L_{N}^{2}} \sqrt{1+L_{N}^{2} \eta^{2}}$, where $a_{\ln }(z)<0$ is the real root number $n+1$ of the equation $M(a, l+1, z)=0$. It is to be noted that the zero $a_{\ln }(z)$ is a monotonic increasing function of $z$ [10, p. 187]. The eigenfrequency follows

$$
\omega=\frac{l}{\left[l+1-2 a_{l n}\left(z_{\eta}\right)\right] \sqrt{1+L_{N}^{2} \eta^{2}}+L_{N}^{2} / 2-1} .
$$

\section{LARGE RADIUS APPROXIMATION}

In the approximation,

$$
z_{\eta} \equiv \frac{R_{0}^{2}}{L_{N}^{2}} \sqrt{1+L_{N}^{2} \eta^{2}} \gg 1
$$

and if $l$ is not very large, then $a_{l n} \approx-n$, and

$$
\begin{aligned}
& \hat{\phi} \propto r^{l} \exp \left(r^{2} \frac{1-\sqrt{1-L_{N}^{2} \eta^{2}}}{2 L_{N}^{2}}\right) \\
& \times M\left(-n, l+1, r^{2} \frac{1-\sqrt{1-L_{N}^{2} \eta^{2}}}{L_{N}^{2}}\right) .
\end{aligned}
$$

and the eigenfrequencies are governed approximately by expression (20) that corresponds to the limit of infinite system. In approximation (27), called a large-radius approximation, the wave properties do not depend on the plasma size.

Condition (27) is a generalization of the one obtained earlier [8] in the case of homogeneous temperature. Now, the large-radius approximation involves the temperature-gradient scale $L_{T}$, and can be satisfied easier. It implies also a less stringent validity condition for the low-frequency approximation. For example, when $L_{N}^{2} \sim 4$ (experimental situations discussed in [8]), the eigenfrequency calculated in the limit of homogeneous temperature approaches the ion-cyclotron frequency, $\omega \sim 0.5 \div 0.8 \Omega_{\mathrm{i}}$, for $l \geq 2, n=0, R_{0}^{2} / L_{N}^{2} \geq 8$, and the usual low frequency approximation breaks down. When the temperature gradient is taken into account, the theory predicts lower eigenfrequencies.

In laboratory applications, the large-radius operation regimes, with their weak dependence on the plasma size $R_{0}$, have advantages, as they can simulate various plasmas.

In the flat density limit, when $L_{N}^{2} \eta^{2} \gg 1$, the condition for the large-radius approximation does not contain the density-gradient scale $L_{N}$ :

$$
R_{0}^{2} / L_{T} \gg 1\left(R_{0}^{2} \gg \rho_{\mathrm{s}} L_{T} \text { in dimensional units }\right) \text {. }
$$

Then the eigenfrequency is governed by expression (22).

Thus the large-radius approximation can be understood within the context of a quantum mechanical analogy. The Schrödinger form of the eigen-value equation shows explicitly that there is a discrete spectrum, and suggest some analytical approximations, the large-radius approximation in particular. According to the boundary condition usual in the quantum-mechanical treatment, the wave function must tend to zero when the radius tends to infinity. Then $M(a, b, z)$ must be a finite polynomial of $z$, which increases slower than the exponential, when $r \rightarrow \infty$. Such a requirement is satisfied if $\left(l+1-E_{\eta}\right) / 2$ is a negative integer, which yields the above result $E_{\eta}=l+2 n+1$.

This requirement is not applicable when the temperature is homogeneous. Then $\tilde{\phi}$ has no exponential asymptotic behaviour at the infinity, and the case of infinite plasma system with $r \rightarrow \infty$ cannot be treated. This seems to be strange. The infinite system can be understood only as the limit $R_{0} \rightarrow \infty$, when a largeradius approximation is appropriate and the "quantummechanical" result is recovered formally.

For the same conditions, the eigen-functions manifest strong localization of the electron density and electric field oscillations near the device boundary [8], where the plasma density and density gradients are the smallest (where there is almost no plasma in fact). Such an expulsion of global oscillations from the plasma core towards the edge without plasma, like in surface-like modes, seems to be strange. It is in contradiction with experimental facts. This observation forces one to take into account some new important and realistic factors such as the radial variation of electron temperature.

\section{NUMERICAL RESULTS: EIGENFREQUENCIES AND GROWTH RATES}

Figure 1 shows the eigenfrequency dependence on $l$, $z_{0} \equiv R_{0}^{2} / L_{N}^{2}$, and $\eta$. With or without temperature gradient, the eigenfrequencies are larger for larger $z_{0}$. In presence of temperature gradient, the eigenfrequency decreases when $\eta$ grows. This decrease is more pronounced for larger $z_{0}$. Therefore, it is easier to observe the temperature-gradient effects in the large-radius regimes (large-size plasmas).

Figures 2-3 show a dependence of the drift-dissipative instability rate $\gamma_{\text {dis }}$ (without axial drift), and of the axialdrift correction $\gamma_{U}$ on the instability rate, on $l$, and $z_{0}$ for some fixed value of $\eta$. Numerical calculations shows that $\gamma_{\text {dis }}$ decreases when $\eta$ grows. As a function of $l$, $\gamma_{\text {dis }}$ has a maximum that shifts to higher $l$ and becomes smaller, as $\eta$ grows. $\gamma_{\text {dis }}$ is also a decreasing function of $L_{N}^{2}$ (this decrease is stronger than in the case of homogeneous temperature, since $\left.\eta=L_{N} / L_{T}\right)$. The dependence of the axial-drift correction $\gamma_{U}$ on $\eta$ is not monotonic. As a function of $l, \gamma_{U}$ has a maximum that shifts to higher $l$ as $\eta$ grows. 


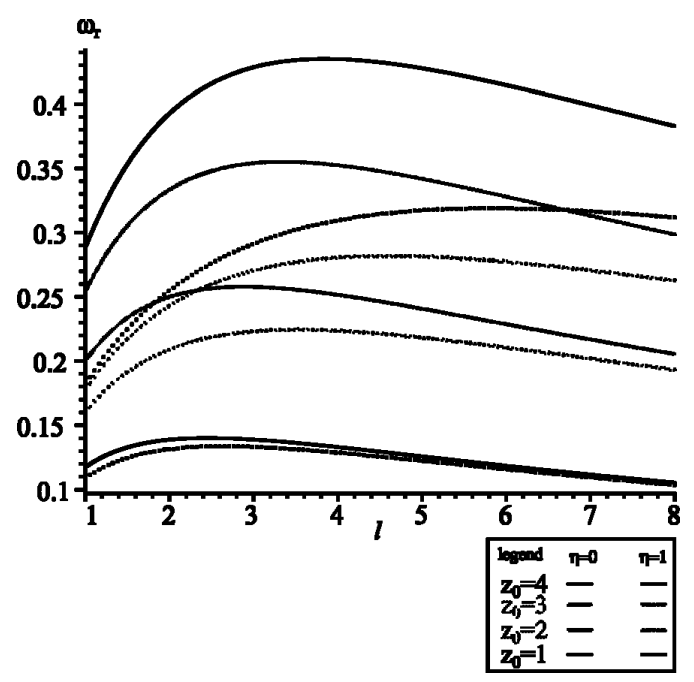

Fig. 1. The eigenfrequency dependence on $l=1 \div 8$, $z_{0}=1 \div 4$, and $\eta$, in the case of Gaussian density profile and Lorentzian temperature profile, for fixed $n=0, L_{N}^{2}=4$; $\eta=0$ (line curves), and $\eta=1$ (dotted curves).

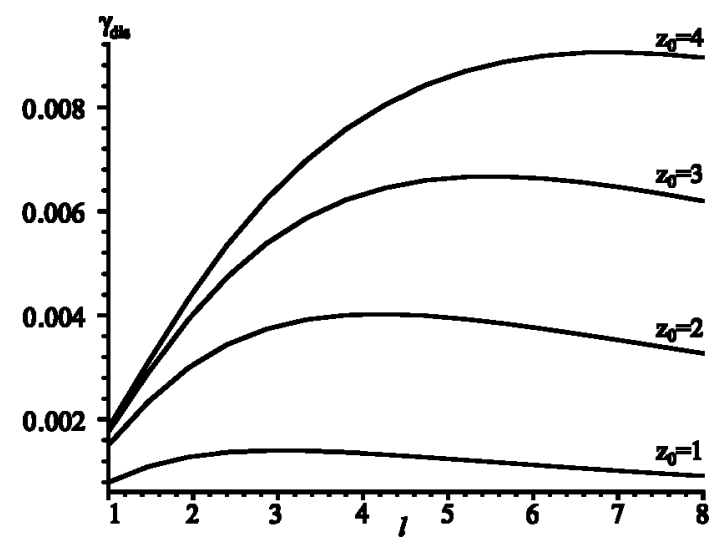

Fig. 2. Weakly non-adiabatic regime $|\delta| \ll 1$, Gaussian density profile and Lorentzian temperature profile: the dependence of the drift-dissipative instability rate $\gamma_{\mathrm{dis}}$ (without axial drift) on $l=1 \div 8$ and $z_{0}=1 \div 4$, for fixed $n=0, \eta=1$, $L_{N}^{2}=4, \Gamma_{\|}=12$. As a function of $l, \gamma_{\text {dis }}$ has a maximum that depends on $z_{0}$. As $z_{0}$ grows, this maximum becomes greater and shifts to higher $l$.

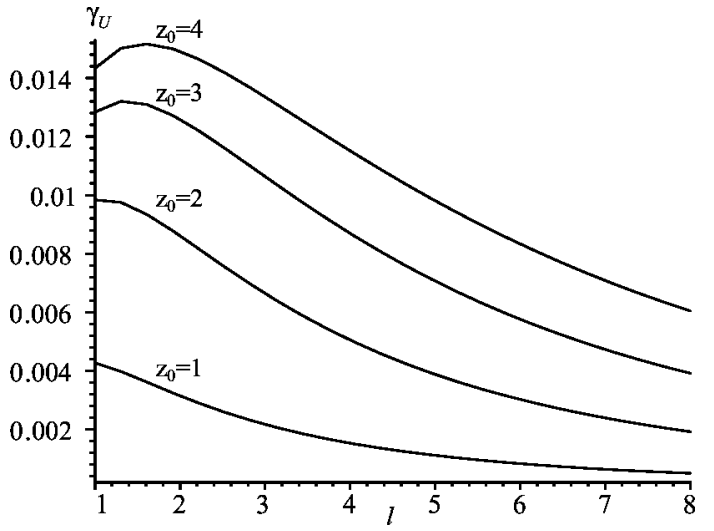

Fig. 3. Weakly non-adiabatic regime $|\delta| \ll 1$, Gaussian density profile and Lorentzian temperature profile: the dependence of the axial-drift correction $\gamma_{U}$ to the instability rate on $l=1 \div 8$ and $z_{0}=1 \div 4$, for fixed $n=0, \eta=1, L_{N}^{2}=4$, $\Gamma_{\|}=12$. As a function of $l, \gamma_{U}$ has a maximum that depends on $z_{0}$. Its position is different from that of $\gamma_{\text {dis }}$. As $z_{0}$ grows, this maximum becomes greater and shifts to higher $l$.

\section{SUMMARY}

In this study we analyzed the properties of global drift waves in the weakly ionized plasma column with macroscopic density and temperature gradients. It is shown that, in the case of adiabatic electrons and for a Gaussian density profile, the problem can be reduced to a Schrödinger's equation. In this regards, the effect of the density and temperature gradient on the "wave function" (i.e., the potential) and the "energy" (and then the eigenfrequency) are explained with the usual quantummechanical arguments. A decreasing profile tends to localize the modes more towards the plasma core and this effect can be accompanied by the eigenfrequency decrease. This effect is enhanced for large size plasmas.

\section{ACKNOWLEDGEMENTS}

The discussions with Drs. A. Zagorodny, Th. Pierre, G. Leclert, G. Bonhomme, G. Mandfredi, A. Ghizzo, and P. Bertrand are very much appreciated.
[1] A. Latten, T. Klinger, A. Piel, Th. Pierre, Rev. Sci. Instrum. 66, 3254 (1995).

[2] U. Kauschke, G. Oelerich-Hill, A. Piel, Phys. Fluids B 2 , № 1,38 (1990).

[3] T. Klinger, A. Latten, A. Piel, G. Bonhomme, Th. Pierre, T. Dudok de Wit, Phys. Rev. Lett. 79, 3913 (1997).

[4] T. Klinger, A. Latten, A. Piel, G. Bonhomme, Th. Pierre, Plasma Phys. Control. Fusion B 39, 145 (1997).

[5] A. Sitenko, V. Malnev, Plasma Physics Theory (Chapman \& Hall, London, Glasgow, Weinheim, New York, Tokyo, Madras, 1995).

[6] R. F. Ellis, Marden-Marshall, Phys. Fluids 22, 2137
(1979).

[7] R. F. Ellis, Marden-Marshall, R. Majeski, Plasma Phys. 22, 113 (1980).

[8] P. P. Sosenko, G. Bonhomme, T. Pierre, J. Plasma Phys. 63, 157 (2000).

[9] B. B. Kadomtsev, Plasma Turbulence (Academic Press, New York 1965).

[10] Buchholz Herbert, The Confluent Hypergeometric Function (with Special Emphsis on its Applications), Springer Tracts in Natural Philosophy. Vol 15. (Springer-Verlag, Berlin-Heidelberg-New York, 1969). 


\title{
КРУПНІ ПЛИННІ ХВИЛІ У СЛАБКОЙОНІЗОВАНОМУ ПЛАЗМОВОМУ СТОВПІ
}

\author{
П. П. Сосенко ${ }^{1,2}$, М. Полені ${ }^{1}$ \\ ${ }^{1}$ Університет Анрі Пуанкаре - Нансі I, \\ Лабораторія фізики йонізованих середовищ та застосувань, \\ ВР 239, 54506, Франція \\ ${ }^{2}$ Мінародний Центр фізики, \\ Інститут теоретичной фізики ім. М.М. Боголюбова НАН Украӥни \\ вул. Метрологічна 14-б, Киӥв, 03143, Украӥна
}

Досліджено вплив теплового перепаду на крупні плинні хвилі у стовпі слабкойонізованої плазми. Показано, що тепловий перепад істотно впливає на властивості хвиль: власну частоту, поріг та швидкість нестійкости, просторовий розподіл. 